\title{
EDITORIAL
}

\section{DISCOVERIES REPORTS: publishing discoveries in medicine, biology and chemistry}

\author{
Octavian Bucur ${ }^{1, *}$ Elisa A. Liehn ${ }^{2, *}$ \\ ${ }^{1}$ Department of Pathology, Harvard Medical School and Beth Israel Deaconess Medical Center, Boston, MA, \\ USA; ${ }^{2}$ Institute for Molecular Cardiovascular Research (IMCAR), RWTH Aachen University, Germany. \\ *Corresponding authors: \\ Octavian Bucur, MD, Department of Pathology, Harvard Medical School and Beth Israel Deaconess Medical \\ Center, 330 Brookline Ave, Boston, MA, 02215, USA. E-mail: obucur@bidmc.harvard.edu
}

Elisa A. Liehn, $M D, P h D$, Institute for Molecular Cardiovascular Research (IMCAR), University Hospital Aachen, Rheinisch-Westfälische Technische Hochschule Aachen, Pauwelsstrasse 30, 52074 Aachen, Germany. Tel.: 0049-241-80 35983; Fax: 0049-241-80 82716; E-mail: eliehn@ukaachen.de

Citation: Bucur O, Liehn EA. DISCOVERIES REPORTS: publishing discoveries in medicine, biology and chemistry. Discoveries Reports 2014, Sep-Dec; 1(1): e4. DOI: 10.15190/drep.2014.4

Discoveries Reports is a peer-reviewed, open access, multidisciplinary online journal, publishing high impact experimental reports, reviews, perspective articles, and editorials from all areas related to medicine, biology and chemistry. These areas include, but are not limited to: Clinical findings, Clinical Trials, Translational Medicine, Cellular and Molecular Biology, Biochemistry, Biophysics, Genomics, Proteomics, Bioinformatics, Systems Biology, Biotechnology, Synthetic Biology, Bioengineering, all areas of Chemistry and Ethics in Science.

Keywords: breakthrough research, interdisciplinary, multidisciplinary, journal, Translational Medicine, Clinical findings, Clinical Trials, Global Medicine, Molecular and Cellular Biology, Biochemistry, Biophysics, Genomics, Proteomics, Systems Biology, Bioinformatics, Biotechnology, Synthetic Biology, Bioengineering, Epidemiology, Organic/ Inorganic/ Physical Chemistry and Ethics in Science

\section{Why launching Discoveries Reports is useful?}

Taken into consideration the tremendous recent advances in scientific discoveries and knowledge, "new high impact platforms for publishing highquality interdisciplinary/multidisciplinary research and cutting edge discoveries are very much needed"1.

Together with Discoveries, Discoveries Reports brings to the research scientific community several innovations that not only will make manuscript's submission and evaluation process faster, but will also set up the groundwork for establishing standard rules of critical reagents validation, in order to address the low reproducibility of the published results highlighted in several key publications ${ }^{2-6}$.

Discoveries Reports is supervised by an outstanding editorial board that aims to implement the following innovations: the authors can submit their manuscript in any format they want (only accepted manuscripts will be modified accordingly), the submission process is quick and efficient (1 minute or less), the Cover Letter is optional, and we support the need of rules for validation of critical reagents (such as antibodies, siRNAs).

Discoveries Reports will also consider a small number of selected experimental articles validating/invalidating highly used reagents in current publications (ex. antibodies). Discoveries Reports will also accept a small number of high impact articles presenting important negative data of wide scientific interest. We think that these 
special types of articles will be of interest for a broad audience, will be highly cited, and will have a significant impact on the validity of the future published research.

Why is important to also publish selected experimental articles validating highly used reagents in current publications?

As previously published ${ }^{1-6}$, "antibodies, other reagents, inadequate animal models or cell-based models/assays, insufficient number of experimental repeats and statistical inadequacies all contribute to the low reproducibility of preclinical and clinical data" 1 .

Since some critical reagents (such as antibodies) already used in publications were found to be insufficiently validated, leading to potential misinterpretations and erroneous conclusions ${ }^{2-6}$, publishing articles which validate selected widely used reagents of critical importance for scientific community is very useful. These manuscripts will not only reveal the best reagents that can be used by scientific community, but will also expose the reagents already used in publications that have been insufficiently validated, thus reducing the published research irreproducibility.

Moreover, the journals and other entities should define adequate rules and measures for reagents validation and their use in publications, in order to decrease irreproducibility of the already published research discoveries ${ }^{1}$. Several publications (such as Cancer Research), have already included rules for validation of critical reagents (such as antibodies) used in submitted manuscripts ${ }^{1,7}$. Thus, Discoveries Reports will aim not only to publish manuscripts describing valuable research undertaken only with validated critical reagents, but will also help the scientific community identifying the insufficiently validated reagents, many of them already used in publications.

Why is important to also publish high impact articles presenting important negative data of wide scientific interest?

The negative data and results most of the time do not get published or reported. Thus, the scientific community may spend valuable time and money performing similar experiments. Moreover, these results may have critical importance in many research areas, including clinical research and clinical trials, which involve human safety. Since not all the negative results are important, our editorial team will carefully evaluate all the manuscripts from this article category to ensure that only the negative data that concerns a broad audience and/or is critical for a scientific field development will be published.

\section{Conflict of Interest}

The authors declare no conflict of interest.

\section{References:}

1. Bucur O, Almasan A, Nikolajczyk BS, Nicolson GL, Lawler L, Velculescu VE et al. Discoveries: an innovative platform for publishing cuttingedge research discoveries in medicine, biology and chemistry. Discoveries 2013, Oct-Dec; 1(1): e1. DOI: 10.15190/d.2013.1

2. Bucur O, Pennarun B, Stancu AL, Nadler M, Muraru MS, Bertomeu T et al. Poor antibody validation is a challenge in biomedical research: a case study for detection of c-FLIP. Apoptosis 2013; 18(10):1154-62.

3. Begley CG, Ellis LM. Drug development: raise standards for preclinical cancer research. Nature 2012; 483(7391): 531-533

4. Prinz F, Schlange T, Asadullah K. Believe it or not: how much can we rely on published data on potential drug targets? Nat Rev Drug Discov. 2011; 10(9): 712

5. Begley CG. Six red flags for suspect work. Nature 2013; 497(7450): 433-4.

6. Mullard A. Reliability of 'new drug target' claims called into question. Nat Rev Drug Discov. 2011; 10(9): 643-644

7. http://www.aacrjournals.org/site/InstrAuthors/if ora.xhtml

DISCOVERIES REPORTS is a peer-reviewed, open access, online, multidisciplinary journal, publishing high impact manuscripts from all areas related to MEDICINE, BIOLOGY and CHEMISTRY; ¿ 2014, Applied Systems 